INRA Prod. Anim., 2004, 17 (3), 237-242
M. VANDEPUTTE, S. LAUNEY

INRA, Génétique des Poissons, F-78352 Jouy en Josas Cedex

Courriel :mvande@jouy.inra.fr

\section{Quelle gestion génétique de la domestication chez les poissons?}

Les poissons occupent une place originale parmi les animaux d'élevage, car la plupart des espèces sont encore peu, voire pas domestiquées. Dans de nombreux cas, les techniques d'élevage sont encore à mettre au point dans le détail, et les cycles d'élevage et de reproduction ne sont qu'imparfaitement bouclés (utilisation fréquente de géniteurs sauvages). De plus, l'aquaculture s'intéresse à de très nombreuses espèces, avec de nombreuses ressources génétiques existant dans la nature, ce qui la différencie aussi des filières d'élevage classiques. La question de la domestication se pose donc fréquemment, d'abord sur un plan très pratique d'échantillonnage et de maintien de la variabilité génétique, nécessaire à un élevage performant. Se pose également la question de la sélection de caractères adaptatifs pour l'élevage et de la manière de l'orienter en fonction des objectifs, qui peuvent être très différents selon que l'on vise par exemple une production intensive de poisson de consommation ou la production d'animaux destinés au repeuplement des cours d'eau.

La question de la domestication des espèces est récurrente en aquaculture, du fait du nombre important de nouvelles espèces pour lesquelles des productions se mettent en place, parfois transitoirement et parfois avec

\section{Résumé}

Depuis 1950, le nombre d'espèces de poissons en production est passée de 42 à 186. La grande diversité des espèces concernées, le caractère récent de la mise en élevage et l'existence de ressources génétiques sauvages pour la plupart des espèces font de la domestication une question récurrente en aquaculture. Les aspects génétiques de la domestication concernent deux aspects majeurs : la phase de mise au point des élevages et de création des souches de départ, et la gestion des populations au cours de l'élevage pour assurer à la fois l'efficacité de la production et le bien-être du poisson. Pour le premier aspect, les études existantes, peu nombreuses, indiquent qu'il est sans doute difficile de conclure sur les aptitudes d'une souche particulière à l'adaptation aux conditions d'élevage sur la simple base de sa variabilité génétique aux marqueurs neutres ou d'observations écologiques en milieu naturel. Un testage de souche peut être pratiqué avant la mise en élevage, mais, pour être pertinent, il doit être réalisé en conditions contrôlées, les plus proches possibles des conditions réelles d'élevage. Dans tous les cas, le choix d'une souche de départ ayant une base génétique la plus large possible et une bonne gestion des reproductions permet d'assurer le maintien de la variabilité génétique au cours des générations. Le processus de domestication s'accompagne de modifications d'un grand nombre de caractères, mais dont les mécanismes génétiques ne sont pas toujours maîtrisés. Il pourrait cependant être possible d'orienter volontairement l'évolution des caractères d'adaptation au milieu d'élevage, à l'aide de programmes de sélection artificielle dirigés. un réel succès. La plupart des espèces aquacoles sont de domestication très récente. Seule la carpe est élevée depuis des siècles, ayant probablement été domestiquée par les Romains (Balon 1995), mais sa domestication reste récente par rapport à celle des autres animaux d'élevage. Le cycle d'élevage de la truite est maîtrisé depuis un peu plus d'un siècle et seules quelques rares espèces aquacoles étaient produites dans la première moitié du $\mathrm{XX}^{\mathrm{e}}$ siècle. Les données disponibles (FAO 2003) montrent en effet qu'en 1950, seules 42 espèces de poissons faisaient l'objet d'une production au niveau mondial, dont 22 d'une production supérieure à 1000 tonnes, et aucune d'une production supérieure à 100000 tonnes. En 1975, 71 espèces étaient produites, dont 43 à plus de 1000 tonnes/an et 7 à plus de 100000 tonnes/an, ces chiffres passant à 186, 88 et 24 en 2001, respectivement. Il faut aussi noter que sur la période 1950-2001, 47 espèces ont fait l'objet d'un début de production pour être abandonnées par la suite. Ces chiffres montrent bien à la fois l'extrême diversité des espèces concernées et le caractère très récent de la domestication de la plupart d'entre elles.

Il faut d'ailleurs remarquer que le terme domestication, appliqué au poisson, signifie souvent pour ceux qui l'emploient la mise au 
point des méthodes d'élevage d'une espèce et la compréhension de ses besoins, devant aboutir à un cycle d'élevage maîtrisé. On pourrait qualifier cette approche de domestication zootechnique, qui est assez différente de la définition génétique généralement admise. En effet, selon Price (1984), la domestication est « le processus par lequel une population d'animaux devient adaptée à l'homme et à l'environnement captif par une ou des combinaisons de modifications génétiques au long des générations et d'événements de développement induits par l'environnement, récurrents à chaque génération ».

Pour bien répondre aux questions qui se posent à l'aquaculture en termes de génétique de la domestication, il nous faut étudier ces deux aspects et déterminer d'abord si la génétique peut permettre de faciliter la phase de mise au point d'une zootechnie de routine, puis de quelle façon gérer les populations pour que leur adaptation à l'élevage se fasse dans les meilleures conditions. Le but est de garantir l'efficacité de la production, tout en assurant le bien-être du poisson en lui permettant d'être bien adapté à son environnement (Vandeputte et Prunet 2002). Dans le cas particulier où l'élevage sert à la production d'animaux pour le repeuplement destiné à la pêche commerciale et de loisir, la question pourra être posée à l'inverse : est-il possible de limiter les modifications induites par la domestication en élevage, et les effets d'une population domestiquée sur les populations naturelles en place sont-ils ou non susceptibles d'être nuisibles ? A l'heure actuelle, l'essentiel des études portant sur les modifications induites par la domestication chez les poissons sont issues de cette dernière problématique.

\section{Peut-on trouver des génotypes $a$ priori favorables à la domestication dans une espèce donnée ?}

Une des particularités des poissons est qu'il existe pour toutes les espèces (à l'exception peut-être de la carpe) des ressources génétiques sauvages, la domestication passe donc souvent par la collecte de géniteurs dans le milieu naturel. Une question souvent posée est de savoir s'il peut exister des génotypes favorisant la domestication zootechnique et/ou dont les performances en élevage pourraient être déduites de leurs caractéristiques en milieu naturel. Il n'est pas simple de répondre à cette question, car une comparaison rigoureuse des génotypes ne peut se faire que toutes choses égales par ailleurs, c'est-àdire en élevage en conditions contrôlées. Les constatations sur les caractéristiques plus ou moins favorables de telle ou telle population ne peuvent être faites qu'a posteriori, une fois le protocole d'élevage au point, et l'on peut dans ce cas parfois faire un rapprochement entre ce qui est observé et les caractéristiques de la population dans son milieu d'origine.

Cependant, d'une manière générale, la comparaison des performances en élevage avec l'écologie des souches en milieu naturel est très peu étudiée, la plupart des résultats de comparaison de souches étant discutés uniquement comme des performances relatives dans les conditions particulières à chaque étude (par exemple : Ayles 1975, Gjedrem 1976, Ferguson et al 1985, Eknath et al 1993). Quand les comparaisons sont faites entre les performances en test et celles dans le milieu d'origine, on voit que ces dernières sont bien peu fiables. Par exemple, lorsque trois souches de carpe françaises (Forez, Brenne et Dombes) ayant des croissances très différentes en apparence (poids commercial de 1,5 à $1,8 \mathrm{~kg}$ atteint en 2 ans en Forez, 3 ans en Dombes et 3-4 ans en Brenne) sont comparées en environnement standardisé, on observe des performances égales entre ces trois souches (Vandeputte et al 2002). Dans le programme de testage norvégien à l'origine de la domestication du saumon atlantique (37 souches testées), la latitude d'origine de la souche ne semble intervenir ni sur la croissance en eau douce, ni sur la smoltification (ensemble des modifications physiologiques et morphologiques précédant la migration en mer), ni sur la croissance en mer, alors même que les performances sont très variables entre souches (Refstie 1977 et 1978, Gunnes et Gjedrem 1978). A contrario, des souches de saumon montrant des différences de date de smoltification en testage proviennent de rivières ayant des caractéristiques semble-t-il très comparables (Nielsen et al 2001).

Un autre indicateur potentiel de performances en élevage est le niveau d'hétérozygotie des souches en milieu naturel. Cependant, s'il est bien certain que la consanguinité fait baisser les performances (Kincaid 1976), il ne semble pas qu'il y ait de façon générale une corrélation entre le niveau d'hétérozygotie d'une souche et sa performance (Chevassus et al 1992), bien que ceci ait pu être décrit dans certains cas (même classement des vitesses d'éclosion et des hétérozygoties dans une comparaison de 3 souches, mais aucune corrélation dans deux autres expériences, Ferguson et al 1985). Ceci peut être dû en partie au fait que si la variabilité réduite résulte d'une sélection, volontaire ou non, l'effet positif de la sélection sur les performances peut masquer l'effet négatif de la consanguinité.

On peut conclure de ce qui précède que les informations disponibles, certes disparates, ne plaident pas en faveur de la possibilité, pour une espèce donnée, de choisir une souche de départ qui aurait des caractéristiques plus favorables qu'une autre en élevage, sur la simple base d'informations sur son écologie ou sa variabilité génétique à des marqueurs neutres. Il n'en reste pas moins que la génétique peut apporter une aide précieuse dans les premières phases du développement de l'aquaculture d'une espèce.

\section{Echantillonner et gérer la variabilité génétique}

En effet, il apparaît de façon assez claire qu'au moins pour les espèces dont l'élevage est maintenant maîtrisé, il existe une variabilité génétique non négligeable des caractères 
zootechniques (croissance, conformation, résistance aux maladies - voir revues de Hulata 1995, Gjedrem 1998, Olesen et al 2003, Vandeputte 2003). Cette variabilité peut être très importante entre souches (par exemple, $56 \%$ d'écart sur le poids à trois ans entre deux souches de carpes, Linhart et al 2002), mais également intra-souche $\left(\mathrm{h}^{2}=0,41\right.$ pour la réponse de stress, Pottinger et Carrick 1999b). De plus, les effets négatifs de la consanguinité sont bien démontrés. Ceci a pour conséquences qu'il peut être intéressant de choisir une souche appropriée pour démarrer un élevage, mais ce choix ne peut se faire que sur la base d'une comparaison en conditions d'élevage et, de fait, des programmes de ce type sont souvent mis en ouvre au démarrage d'une filière de production (par exemple Gunnes et Gjedrem (1978) pour le saumon atlantique, Bentsen et al (1998) pour le tilapia aux Philippines, Chevassus et al (1992) pour la truite commune en France). Même dans les cas où le choix d'une souche après un programme de testage n'est pas possible, il est essentiel, pour fonder une souche d'élevage, d'utiliser un nombre suffisant de géniteurs pour avoir une base génétique la plus large possible (Chevassus 1989), et ceci pour au moins quatre raisons : - pour éviter de développer rapidement de la consanguinité en étant amené à recroiser rapidement entre eux des animaux fortement apparentés ;

- pour avoir dans la population des gènes « rares » contrôlant des caractères qui seraient fortement contre-sélectionnés dans le milieu naturel mais qui auraient un impact positif en élevage (par exemple l'absence de fuite devant un prédateur - voir plus loin) ; - pour avoir une population représentative de la population de départ, ce qui peut être important si la population de départ a des performances intéressantes que l'on souhaite conserver, ou si l'on veut utiliser la population d'élevage comme base pour le repeuplement du stock sauvage d'origine ;

- pour maintenir une variabilité génétique suffisante pour permettre l'amélioration par sélection artificielle.

Les préconisations pour maximiser la part de variabilité génétique transmise à la génération suivante sont d'utiliser un nombre important de reproducteurs à chaque génération, d'utiliser si possible autant de mâles que de femelles, de veiller à l'équi-représentation des parents dans chaque génération de descendants et, si possible, d'éviter les croisements par couples au profit des croisements de type factoriel (Chevassus 1989, Dupont-Nivet et al 2004). Ceci permet d'éviter la dégradation des performances liée à la consanguinité. Celle-ci peut en effet annuler tous les effets bénéfiques de la sélection volontaire ou involontaire de génotypes adaptés à l'élevage, comme dans le cas du tilapia en Asie où des souches sauvages sont plus performantes que les souches d'élevage (Eknath et al 1993), ou dans celui de la carpe où cinq générations de sélection sur la croissance ont abouti à une lignée moins performante (-8\%) que le témoin (Moav et Wohlfarth 1976).

En pratique, ces recommandations sont lourdes à mettre en œuvre puisqu'il est cou- ramment admis qu'il faut entre 50 et 200 reproducteurs (25 à 100 par sexe) participant effectivement à la constitution de la génération suivante pour conserver la variabilité génétique d'une population. Cependant, si ces recommandations sont essentielles dans le cas d'un stock fermé, l'introduction régulière de migrants venus d'autres populations (qui est fréquente dans la pratique aquacole) permet de limiter fortement l'accroissement de consanguinité et la perte de variabilité sans utiliser de grands nombres de géniteurs. C'est sans doute ce qui a permis aux stocks domestiques de truite commune en France d'avoir une variabilité moyenne meilleure que les stocks sauvages dont ils sont issus (Chevassus et al 1992). Cependant, de telles introductions pourront également perturber la sélection volontaire ou involontaire lors de la domestication.

\section{Les mécanismes de la domestication et leurs effets sur l'animal}

Au-delà de recommandations de bonne gestion de la variabilité génétique, il est intéressant de voir quels mécanismes entrent en jeu pour arriver à une réelle domestication. Les effets de la domestication sur les poissons ont été décrits dans plusieurs revues (Ruzzante 1994, Gross 1998, Vandeputte et Prunet 2002) et sont principalement une croissance accrue, une perte du comportement anti-prédateurs et des perturbations de la reproduction (perte des comportements de cour, rétention des ovules et du sperme), modifications qui sont d'ailleurs rencontrées dans la plupart des espèces animales domestiquées. Des variations d'agressivité sont aussi notées, mais dans des sens contradictoires selon les expérimentations - le bénéfice d'être plus ou moins agressif dépendant de la relation entre agressivité et accès à la nourriture, qui est variable selon les conditions d'élevage (Ruzzante et Doyle 1991, Ruzzante 1994).

Les mécanismes génétiques de la domestication peuvent être de quatre types :

- consanguinité et dérive génétique, comme nous venons de le voir. Cependant, si ceci peut aboutir à une divergence par rapport aux populations naturelles, cette modification n'est pas orientée (positivement ou négativement) dans le cas de la dérive génétique. Au contraire, la consanguinité a généralement des conséquences négatives sur la productivité ;

- « sélection naturelle » en captivité, c'est-àdire une sélection involontaire liée à l'environnement d'élevage. Par exemple, la sélection involontaire représente près du tiers du gain obtenu dans une population de saumon sélectionnée pour la croissance à partir d'une souche sauvage (Hershberger et al 1990). Il est également vraisemblable que les perturbations du comportement reproducteur observées chez les salmonidés (Fleming et al 2000, par exemple) soient dues en partie au fait que les animaux pondant spontanément dans les structures 
d'élevage soient voués à une mort génétique en ne participant pas à la création de la génération suivante (n'étant de fait plus disponibles pour la reproduction artificielle, qui est la règle chez les salmonidés). Enfin, il faut noter que cette sélection semble très forte surtout lors de la première génération de reproduction en captivité ( Doyle $e t a l$ 1995) ;

- relâchement de la sélection naturelle, ce qui pourrait expliquer en partie la perte du comportement antiprédateur, qui a un coût (perte de possibilités d'alimentation, stress intense et ses conséquences physiologiques) mais est puissamment sélectionné en milieu naturel, ce qui n'est pas le cas en élevage. Ceci pourrait aussi expliquer en partie les pertes de comportement reproducteur chez les salmonidés, ces comportements étant inutiles quand la reproduction se fait in vitro ;

- sélection artificielle enfin, principalement appliquée en pratique pour la vitesse de croissance, qui est presque toujours en faveur des domestiques dans les comparaisons sauvages-domestiques.

Il faut y ajouter les effets d'environnement récurrents qui modifient le phénotype des animaux d'élevage (par exemple, les saumons d'élevage sont plus gras et ont des nageoires plus petites que les saumons sauvages : Gross 1998).

La domestication de la même espèce dans des milieux très contrastés peut conduire à la sélection de caractéristiques très différentes. Par exemple, les souches de carpe d'origine chinoise auraient une maturité sexuelle plus précoce, une taille moyenne moindre et une plus grande aptitude à l'échappement que les souches européennes, du fait du système d'élevage en Chine (pêche au filet sans vidange des étangs, les reproducteurs étant les animaux qui restent dans l'étang après pêche) très différent du système européen, où les étangs sont pêchés par vidange et les reproducteurs choisis parmi les plus «beaux » poissons (Wohlfarth et al 1975).

Ces mécanismes déterminent la domestication telle que nous pouvons l'observer chez les poissons. Cependant, à part pour la croissance, ces modifications sont en grande partie involontaires et l'on peut se demander s'il ne serait pas possible de les orienter, pour avoir des animaux réellement adaptés à l'élevage.

\section{Que peut faire la sélection artificielle ?}

Si le premier caractère sélectionné en élevage est la vitesse de croissance, de très nombreux autres présentent une variabilité génétique additive inter ou intra-souche. Parmi ceux-ci, on trouve d'autres caractères « classiques » ayant un impact économique direct, comme la qualité de la chair (voir par exemple : Kause et al (2002) pour les rendements de transformation, la teneur en gras, en protéines, en eau et la couleur), la résistance aux maladies (par exemple : Henryon et al 2002), l'âge à la puberté et la date de ponte (par exemple : Su et al 1999). On trouve aussi, de façon plus fragmentaire, des caractères d'adaptation au milieu et de comportement, comme la tolérance aux eaux acides (Gjedrem 1976) ou à de hautes (Perry et al 2001) ou basses (Babouchkine 1987) températures, le comportement agressif (Ruzzante et Doyle 1991, Vollestad et Quinn 2003), la réponse de stress (Pottinger et Carrick 1999a, Tanck et al 2001, Fevolden et al 2002). Pour la plupart, les études montrent juste l'existence de variabilité génétique, parfois suivie par la mise en place d'une sélection expérimentale. Mais aucun de ces caractères d'adaptation ou de comportement ne fait ou n'a fait l'objet de sélection à but commercial, à part la résistance au froid chez la carpe qui a donné une souche « Ropsha » largement utilisée en Europe de l'Est (Babouchkine 1987). Ces caractères, sans impact économique directement mesurable dans la plupart des cas, peuvent cependant très certainement avoir un impact sur la facilité d'élevage des animaux et sur l'amélioration de leur bien-être, qui est une préoccupation grandissante.

\section{Conclusion : quelles straté- gies sont envisageables ?}

La gestion génétique de la domestication est une question à multiples facettes et, dans bien des cas, les informations disponibles sont trop fragmentaires pour pouvoir tirer des conclusions définitives. Il semble par exemple difficile de choisir a priori des génotypes favorables à la domestication au sein d'une espèce sans les avoir testés auparavant, mais le nombre d'expérimentations sur lesquelles cette réponse est bâtie reste très limité. Un apport certain de la génétique dans la domestication d'une espèce est la possibilité de mettre en place des stratégies d'échantillonnage et de maintien de la variabilité génétique, qui sont toujours positives sur le long terme en permettant l'évolution du stock sous l'effet de pressions sélectives, qu'elles soient maîtrisées ou involontaires.

On peut aussi constater l'existence d'une sélection de domestication chez les poissons, mais elle reste non maîtrisée pour l'instant. Pour en tirer le meilleur parti, il semble raisonnable de recommander de domestiquer les animaux dans un système d'élevage représentatif de la réalité de la production, pour permettre une adaptation du stock aux conditions d'élevage réelles. Par ailleurs, l'existence de variabilité génétique démontrée pour des caractères d'adaptation et de comportement laisse une porte ouverte à une sélection volontaire pour ce type de caractères, afin d'accentuer ou d'accélérer la domestication ou, à l'inverse, de la contrarier dans le cas de stocks destinés au repeuplement. Cependant, la mesure directe de ces critères est souvent lourde et le coût d'une telle sélection serait vraisemblablement élevé. Sa mise en œuvre pratique nécessiterait, d'une part, de pouvoir évaluer le gain d'efficacité par rapport à la sélection de domestication involontaire et, d'autre part, de développer des critères de mesure simples et fiables, corrélés génétiquement aux caractères d'intérêt. 


\section{Références}

Ayles G.B., 1975. Influence of the genotype and the environment on growth and survival of rainbow trout (Salmo gairdneri) in central Canadian aquaculture lakes. Aquaculture, 6, 181-188.

Babouchkine Y.P., 1987. La sélection d'une carpe résistant à l'hiver. In : Proc. World Symp. on Selection, Hybridization, and Genetic Engineering in Aquaculture, vol. 1, 447-454, Bordeaux (France) 27-30 May 1986. Heenemann Verlagsgesellschaft mbH, Berlin.

Balon E.K., 1995. Origin and domestication of the wild carp, Cyprinus carpio: from Roman gourmets to the swimming flowers. Aquaculture, 129, 3-48.

Bentsen H.B., Eknath A.E., Palada-de-Vera M.S., Danting J.C., Bolivar H.L., Reyes R.A., Dionisio E.E., Longalong F.M., Circa A.V., Tayamen M.M., Gjerde B., 1998. Genetic improvement of farmed tilapias: growth performance in a complete diallel cross experiment with eight strains of Oreochromis niloticus. Aquaculture, 160, 145-173.

Chevassus B., 1989. Aspects génétiques de la constitution de populations d'élevage destinées au repeuplement. Bull. Fr. Pêche. Piscic., 319, 146-168.

Chevassus B., Krieg F., Guyomard R., Blanc J.M., Quillet E., 1992. The genetics of brown trout: twenty years of French research. Buvisindi Icel. Agric. Sci., 6, 109-124.

Doyle R.W., Herbinger C.M., Taggart C.T., Lochmann S., 1995. Use of DNA microsatellite polymorphism to analyse genetic correlations between hatchery and natural fitness. Am. Fish. Soc. Symp., 15, 205-211.

Dupont-Nivet M., Vandeputte M., Haffray P., Chevassus B. 2004. Efficacité de différents plans d'accouplement pour la conservation de la variabilité génétique dans des populations de poissons. In : Actes du 4e colloque national du BRG, La Chatre, 14-16 octobre 2002, France, sous presse.

Eknath A.E., Tayamen M.M., Palada-de-Vera M.S., Danting J.C., Reyes R.A, Dionisio E.E, Capili J.B, Bolivar H.L., Abella T.A., Circa A.V., Bentsen H.B., Gjerde B., Gjedrem T., Pullin R.S.V., 1993. Genetic improvement of farmed tilapias : the growth performance of eight strains of Oreochromis niloticus tested in different farm environments. Aquaculture, 111, 171-188.

FAO, 2003. Aquaculture production 2001. FAO Yearbook of Fisheries Statistics, 92/2.

Ferguson M.M., Danzmann R.G., Allendorf F.W., 1985. Developmental divergence among hatchery strains of rainbow trout (Salmo gairdneri). I. Pure strains. Can. J. Genet. Cytol., 27, 289-297.

Fevolden S.E., Roed K.H., Fjalestad K.T., 2002. Selection response of cortisol and lysozyme in rainbow trout and correlation to growth. Aquaculture, 205, 61-75.

Fleming I.A., Hindar K., Mjolnerod I.B., Jonsson B., Balstad T., Lamberg A., 2000. Lifetime success and interactions of farm salmon invading a native population. Proc. Roy. Soc. B, 267, 1517-1523.

Gjedrem T., 1976. Genetic variation in tolerance of brown trout to acid water. SNSF Project, Norway, FR5/76, 11 p

Gjedrem T., 1998. Developments in fish breeding and genetics. Acta Agr. Scand. A Anim. Sci., Suppl. 28, 19-26.

Gross M.R., 1998. One species with two biologies: Atlantic salmon (Salmo salar) in the wild and in aquaculture. Can. J. Fish. Aquat. Sci., 55 (Suppl. 1), 131-144.

Gunnes K., Gjedrem T., 1978. Selection experiments with salmon. IV. Growth of Atlantic salmon during two years in the sea. Aquaculture, 15, 19-33.

Henryon M., Jokumsen A., Berg P., Lund I., Pedersen P.B., Olesen N.J., Slierendrecht W.J., 2002. Genetic variation for growth rate, feed conversion efficiency, and disease resistance exists within a farmed population of rainbow trout. Aquaculture, 209, 59-76.

Hershberger W.K., Myers J.M., Iwamoto R.N., McCauley W.C., Saxton A.M., 1990. Genetic changes in the growth of coho salmon (Oncorhynchus kisutch) in marine-net pens, produced by ten years of selection. Aquaculture, 85, 187-197.

Hulata G., 1995. A review of genetic improvement of the common carp (Cyprinus carpio L.) and other cyprinids by crossbreeding, hybridization and selection. Aquaculture, 129, 143-155.
Kause A., Ritola O., Paananen T., Mantysaari E., Eskelinen U., 2002. Coupling body weight and its composition: a quantitative genetic analysis in rainbow trout. Aquaculture, 211, 65-79.

Kincaid H.L., 1976. Inbreeding in rainbow trout (Salmo gairdneri). J. Fish. Res. Board Can., 33, 2420-2426.

Linhart O., Gela D., Rodina M., Slechtova V., Slechta V., 2002. Top-crossing with paternal inheritance testing of common carp (Cyprinus carpio L.) progeny under two altitude conditions. Aquaculture, 204, 481-491.

Moav R.,.Wohlfarth G.W., 1976. Two way selection for growth rate in the common carp (Cyprinus carpio L.). Genetics, 82, 83-101.

Nielsen C., Holdensgaard G., Petersen H.C., Björnsson B.T., Madsen S.S., 2001. Genetic differences in physiology, growth hormone levels and migratory behaviour of Atlantic salmon smolts. J. Fish Biol., 59, 28-44.

Olesen I., Gjedrem T., Bentsen H.B., Gjerde B., Rye M. 2003. Breeding programmes for sustainable aquaculture. J. Appl. Aquac., 13, 179-204.

Perry G.M.L., Danzmann R.G., Ferguson M.M., Gibson J.P., 2001. Quantitative trait loci for upper thermal tolerance in outbred strains of rainbow trout (Oncorhynchus mykiss). Heredity, 86, 333-341.

Pottinger T.G.,.Carrick T.R., 1999a. A comparison of plasma glucose and plasma cortisol as selection markers for high and low stress-responsiveness in female rainbow trout. Aquaculture, 175, 351-363.

Pottinger T.G...Carrick T.R., 1999b. Modification of the plasma cortisol response to stress in rainbow trout by selective breeding. Gen. Comp. Endocr., 116, 122-132.

Price E.O., 1984. Behavioral aspects of animal domestication. Q. Rev. Biol., 59, 1-32.

Refstie T., 1977. Selection experiments with salmon. II. Proportion of Atlantic salmon smoltifying at 1 year of age. Aquaculture, 10, 231-242.

Refstie T., 1978. Selection experiments with salmon. III. Genetic and environmental sources of variation in length and weight of Atlantic salmon in the freshwater phase. Aquaculture, 14, 221-234.

Ruzzante D.E., 1994. Domestication effects on aggressive and schooling behaviour in fish. Aquaculture, 120, 1-24.

Ruzzante D.E.,.Doyle R.W., 1991. Rapid behavioura changes in medaka (Oryzias latipes) caused by selection for competitive and noncompetitive growth. Evolution, 45, 1936-1946.

Su G.S., Liljedahl L.E., Gall G.A.E., 1999. Estimates of phenotypic and genetic parameters for within-season date and age at spawning of female rainbow trout. Aquaculture, $171,209-220$.

Tanck M.W.T., Vermeulen H., Bovenhuis K., 2001. Heredity of stress-related cortisol response in androgenetic common carp (Cyprinus carpio L.). Aquaculture, 199, 283294.

Vandeputte M., 2003. Selective breeding of quantitative traits in the common carp (Cyprinus carpio L.): a review. Aquat. Liv. Resour., 16, 399-407.

Vandeputte M.,.Prunet P., 2002. Génétique et adaptation chez les poissons : domestication, résistance au stress et adaptation aux conditions de milieu. INRA Prod. Anim., 15, 365-371.

Vandeputte M., Peignon E., Vallod D., Haffray P., Komen J., Chevassus B., 2002. Comparison of growth performances of three French strains of common carp (Cyprinus carpio) using hemi-isogenic scaly carp as internal control. Aquaculture, 205, 19-36.

Vollestad L.A., Quinn T.P., 2003. Trade-off between growth rate and aggression in juvenile coho salmon, Oncorhynchus kisutch. Anim. Behav., 66, 561-568.

Wohlfarth G.W., Moav R., Hulata G., 1975. Genetic differences between the Chinese and European races of the common carp. II. Multi-character variation - a response to the diverse methods of fish cultivation ine Europe or China. Heredity, 34, 341-350. 


\section{Abstract}

The genetic management of fish domestication

Since 1950, the number of fish species used in aquaculture has increased from 42 to 186 . Due to this great diversity, to the fact that most species have been bred for a short time, and to the existence of wild genetic resources, domestication is a recurring question in aquaculture development. Genetic aspects of domestication relate to two main aspects : the initial setup of breeding techniques and creation of stocks, and the management of populations across generations, to provide both production efficiency and animal welfare. Concerning the first aspect, the scarce existing studies show that it might be difficult to draw conclusions regarding the ability of a particular population to adapt to a captive environment, based only on genetic variability at neutral markers or on ecological obser- vations in the wild. A strain evaluation program may be conducted prior to the start of the breeding program. It must however be done under controlled conditions, as close as possible to the future breeding conditions. In any case, it is advisable to start the domestication process from a population with a large genetic basis, and to manage it in order to maintain suitable levels of genetic variability across generations. The domestication process leads to important changes in many physical, ethological and/or physiological traits, but the genetic mechanisms of these changes are not always controlled. It could be however possible to direct the evolution of adaptive traits, through targeted selective breeding programs.

VANDEPUTTE M., LAUNEY S., 2004. Quelle gestion génétique de la domestication chez les poissons ? INRA Prod. Anim., 17, 237-242. 